\title{
Molecular (pathologic) markers of tumor invasiveness $=$ more or less (clinical) malignant adenocarcinomas? Wait a second, we are not there yet!
}

\author{
Dao M. Nguyen, MD, MSc, FRCSC, FACS, and Nestor Villamizar, MD
}

\author{
From the Thoracic Surgery Section, Division of Cardiothoracic Surgery, The DeWitt Daughtry Department of \\ Surgery, University of Miami, Miami, Fla. \\ Disclosures: Authors have nothing to disclose with regard to commercial support. \\ Received for publication June 21, 2018; revisions received June 21, 2018; accepted for publication June 22, 2018. \\ Address for reprints: Dao M. Nguyen, MD, MSc, FRCSC, FACS, 1295 NW 14th St, Suite J, Miami, FL 33125 \\ (E-mail: dnguyen4@med.miami.edu). \\ J Thorac Cardiovasc Surg 2018;156:1689-90 \\ $0022-5223 / \$ 36.00$ \\ Copyright (C) 2018 by The American Association for Thoracic Surgery \\ https://doi.org/10.1016/j.jtcvs.2018.06.077
}

Primary lung adenocarcinomas are pathologically heterogeneous, consisting of many subtypes (acinar, papillary, micropapillary, and solid) with established prognostic significance. $^{1,2}$ Radiographically, early stage (T1) adenocarcinomas frequently present either as a solid tumor or as a component of part-solid ground-glass opacity (GGO). The treatment of the former is straightforward, with anatomic resection and systemic thoracic lymphadenectomy being the standard of care (lobectomy or in properly selected cases-favorable anatomic location, tumor size $\leq 2 \mathrm{~cm}$, borderline pulmonary reserve-segmentectomy with sufficiently wide parenchyma surgical margin). The management of GGO-associated adenocarcinoma is more nuanced. ${ }^{3}$ There is little controversy that surgical resection is indicated for part-solid GGO, because the solid component is invasive cancer nested within the noninvasive lepidic adenocarcinoma (lepidic-positive adenocarcinoma). The magnitude of lung resection is influenced by tumor characteristics (sizes and the proportions of solid component within the GGO, consolidation tumor ratio) as well as patients' underlying conditions. Moreover, lepidic-positive adenocarcinomas have better overall survival outcomes than those without a radiographic or pathologic lepidic component (lepidic-negative adenocarcinomas). ${ }^{4}$ The pathobiologic basis of such differential clinical behavior has not been fully elucidated.

In a translational research study published in the current issue of the Journal, Katsumata and colleagues ${ }^{5}$ (Departments of Surgery and Pathology, National Cancer Center Hospital, Kashiwa, Japan), hypothesized that "the tumor microenvironment of the invasive component of [lepidicpositive] adenocarcinoma would differ from that of [lepidic-negative] adenocarcinoma, and that this relationship would persist in lesions with size-matched invasive (nonlepidic) components." They performed comparative analysis of expression of cancer stem-cell related markers

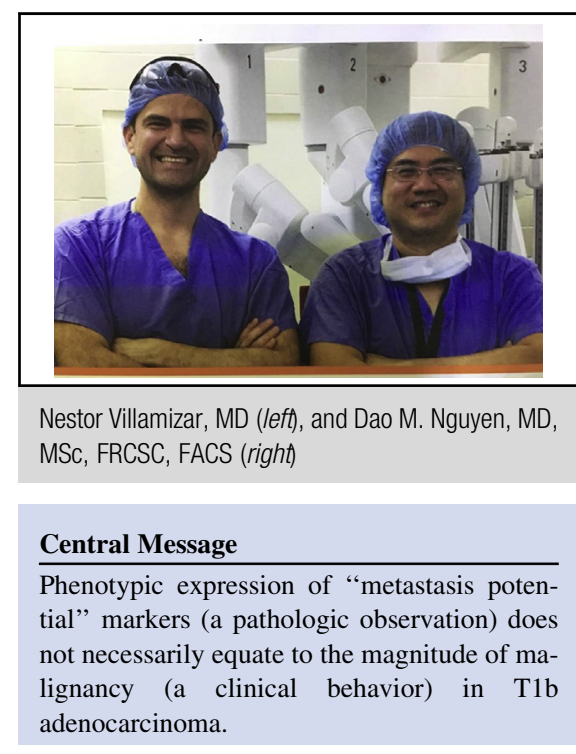

See Article page 1679 .
(ALDH1 and CD44), invasion-related marker (laminin-5) and hypoxia-related markers (GLUT1 and CAIX) and tumor-promoting stromal cells PDPN-positive cancerassociated fibroblasts and $\mathrm{CD}_{20}{ }^{+}$tumor-associated macrophages in size-matched (T1b 1 to $2 \mathrm{~cm}$ ) invasive component of lepidic-negative and lepidic-positive pathologic stage 1A adenocarcinomas resected by lobectomy and thoracic lymphadenectomy. Although lepidic-positive adenocarcinomas, as a group and only according to statistical analysis, express modestly lower levels of cellular markers known to be associated with "invasion and metastasis potential" when compared with lepidicnegative counterparts, there is absolutely no difference in pathologic evidence of lymphatic and vascular invasion between the groups. More strikingly, there is no statistically difference in overall and recurrence-free survivals of the cohorts. The differential phenotypic expression profiles of selected markers of "invasiveness" and of "metastasispromoting stromal cells" in the invasive component of lepidic-positive and lepidic-negative adenocarcinomas are of pathobiologic interest (representing "more or less malignant microenvironment") but not validated by clinical data. They cannot by "a leap of faith" be equated with differential clinical outcomes and be used to support the 
"suggestion" by Katsumata and colleagues, ${ }^{5}$ "Given that $\mathrm{Lep}^{+}$[lepidic-positive] adenocarcinoma is less invasive [phenotypically but not pathologically or clinically] with less metastatic potential [speculating from the expression profile of molecular markers], early stage small $\mathrm{Lep}^{+}$ [lepidic-positive] adenocarcinoma may be treated with limited resection techniques."

The findings by Katsumata and colleagues ${ }^{5}$ are sufficiently compelling to warrant more in-depth clinical analysis. The strength of their study is its hypothesis-driven and novel approach of cellular and molecular analysis of size-matched invasive components of 2 pathologically diverse lung adenocarcinomas. The main weakness of this article is the total lack of sufficient clinical data to support the statements in the central picture legend and in the perspective statement. One has to exercise extreme caution in translating pathologic observations to tumor biology and malignant behavior without proper correlative clinical outcomes. What surgeons need to guide their decision on the magnitude of lung resection for lepidic-positive adenocarcinoma is clinical demonstration of a more indolent clinical course and better outcomes in this population, not immunohistochemical staining of some cellular and molecular markers.

\section{References}

1. Solis LM, Behrens C, Raso MG, Lin HY, Kadara H, Yuan P, et al. Histologic patterns and molecular characteristics of lung adenocarcinoma associated with clinical outcome. Cancer. 2012;118:2889-99.

2. Kamiya K, Hayashi Y, Douguchi J, Hashiguchi A, Yamada T, Izumi Y, et al. Histopathologic features and prognostic significance of the micropapillary pattern in lung adenocarcinoma. Mod Pathol. 2008;21:992-1001.

3. Suzuki K. Whack-a-mole strategy for multifocal ground glass opacities of the lung. J Thorac Dis. 2017;9(Suppl 3):S201-7.

4. Hattori A, Matsunaga T, Takamochi K, Oh S, Suzuki K. Prognostic impact of a ground glass opacity component in the clinical $\mathrm{T}$ classification of non-small cell lung cancer. J Thorac Cardiovasc Surg. 2017;154:2102-10.

5. Katsumata S, Aokage K, Miyoshi T, Tane K, Nakamura H, Sugano M, et al. Differences in tumor microenvironment between stage I lepidic-positive and lepidic-negative lung adenocarcinoma. J Thorac Cardiovasc Surg. 2018;156: 1679-88. 